\title{
Improving the specific instrumental transversal skill of Aerospace Engineers through a lab experience
}

\author{
Ricardo Novella ${ }^{1}$, Jorge García-Tíscar ${ }^{1}$, Carlos Micó ${ }^{1}$, Josep Gomez-Soriano ${ }^{1, *}$ \\ ${ }^{1} \mathrm{CMT}$ - Spain
}

\begin{abstract}
This paper proposes an educational methodology for the development of the specific instrumental transversal skill in Aerospace Engineering degree students. This methodology uses an autonomous and active learning approach to measure, through two different techniques, the local flow velocities around a circular cylinder in a subsonic wind tunnel. The students are directly involved in the complete measuring procedure through practical sessions in a lab, applying directly Hot-Wire Anemometry (HWA) and Particle Image Velocimetry (PIV) techniques, and reporting the analysis of the results after the corresponding data processing. Results show that the proposed lab-based methodology is well received by the students whereas the objective evaluation demonstrates its potential to improve the specific instrumental transversal skill acquisition.
\end{abstract}

Keywords: Transversal skills, Active learning, Autonomous learning, Aerospace Engineering, Fluid mechanics

\section{Introduction}

Spain was one of the early adopters of the European Higher Education Area (EHEA), so the degrees in the Spanish state are already fully integrated into this common framework (European Ministers of Education, 1999). One of the most relevant novelties arising from this integration is the clear trend towards the progressive implementation of continuous assessment systems (Carrillo de la Peña \& Pérez, 2012) and active learning methodologies (Freeman et al., 2014; Lombardi \& Oblinger, 2007) due to their undoubtable benefits over the learning process. Additionally, the curricula are being adjusted to align up to a given extent with the original (Bloom, 1956) or revised (Marzano \& Kendall, 2006) Bloom' taxonomy classification. Thus, the degrees in general and those related to Engineering in particular focus the learning skills on the knowledge and comprehension in the introductory 
years, transitioning to the synthesis and evaluation as the students reach the final years. Here the students are expected to solve real problems involving design and project development activities. As a result, the learning methodology needs to be adapted.

In agreement with the previous European and Spanish educative scheme, the University where the authors develop their academic career, Universitat Politècnica de València, prove its commitment with the ESHE by designing and implementing an institutional educative long-term project by which the thirteen transversal skills described in Section 2 will be developed and evaluated in all its degrees (UPV, 2015).

In the case of the Aerospace Engineering degree, the transversal skill designed as Specific Instruments, which devotes to the use and development of materials and methods, is of great relevance considering the advanced experimental techniques and methodologies applied in this field that are usually not accessible for the students (Lapins, 1997).

In this framework this paper describes a suitable learning methodology already implemented in an Aerospace Engineering degree course dedicated to the development of the previous transversal skill. In detail, two of the most extensively applied experimental techniques to measure velocities in fluid flows are introduced to the students through practical sessions in a laboratory environment. Following an active and autonomous learning approach, the students are directly involved in the complete measuring sequence, applying directly these techniques, known as Hot-Wire Anemometry (HWA) and Particle Image Velocimetry (PIV), and reporting the analysis of the results after the corresponding data processing.

\section{Course and skill description}

The details of the course entitled Testing in Propulsive Systems included in the Aerospace degree curriculum, in which the learning methodology is implemented, are described in this section with the aim of defining the context.

This course is offered to the students as one of the alternatives in the set of optative courses included in the specific technologies' portfolio. The course develops in the first (Autumm) semester of the fourth (last) year and consist of 4.5 credits ECTS (European Credits Transder System), which corresponds to 45 hours of presential plus 67.5 hours of nonpresential learning activities. The average number of students is 30 (83\% males and $17 \%$ females) and fluctuates depending on the academic year between 27 and 33 and thus, the population is expected to be representative for further analysis of the learning results. 
As a short description, this course provides the foundations of the design and development of experimental methodologies for the study of the thermo-fluid-dynamics phenomena in propulsive systems with application to the aerospace field. According to this target, the first unit introduces the theoretical framework of the experimental techniques applied to this field, the second and the third units discuss the most relevant intrusive and non-intrusive experimental techniques respectively.

This paper focuses on the specific instruments transversal skill, which refers to the use of the tools and technologies required for the future professional career of the student depending on the given degree. Thus, after being trained in this transversal skill the student will identify the most suitable tools according to the nature of the targeted activity, integrating and combining them efficiently to design a dedicated experiment, solve a problem or develop a complete project.

Then, the designed methodology promotes the development of the previous transversal skill by involving the students in more or less extent on the complete measuring chain, including (1) experimental setup configuration, (2) testing and data acquisition, (3) calibration of the measuring equipment, (4) data analysis and (5) synthesis and report of the results

Thus, the idea is to generate an active and autonomous learning methodology where the students reproduce the hands-on and decision making environment that they later will face along their professional career. To this end, six different lab sessions are held, three regarding intrusive measurement techniques (pressure measurement, temperature measurement, HWA) and three focused on optical techniques (Schlieren, PIV and Laser Doppler Anemometry). The work presented here aims to link both intrusive and optical approaches by analyzing the same flow setup in the HWA and PIV experiences, allowing the students to perform the five aforementioned steps of the measurement chain for both methods.

\section{Lab session materials and methods}

\subsection{Experimental facility}

The activities consisted on the application of two experimental techniques to the same case study: the characterization of the steady flow in the wake of a circular cylinder. The flow around a circular cylinder has been broadly studied both as validation case and as a legitimated research case. The flow around a cross-sectional cylinder is steady and symmetrical at low Reynolds numbers. Increasing the Reynolds number, asymmetries appear, eventually resulting in the Von Karmann vortex street, and on to a fully-developed 
Improving the specific instrumental transversal skill of Aerospace Engineers through a lab experience

turbulent wake. In this sense, it is an extremely complete case to observe and analyze multiple flow behaviors in only one configuration.

\subsection{Hot-Wire Anemometry}

This method is based on the principle that the electrical resistance of multiple metals is a function of temperature. Taking advantage of the existence of this relationship, a wire that is pre-heated when an electric current passes through it is introduced into the flow. Since the flow tends to cool down the wire due to convection (other aspects could be considered negligible), the wire temperature directly depends on the flow speed.
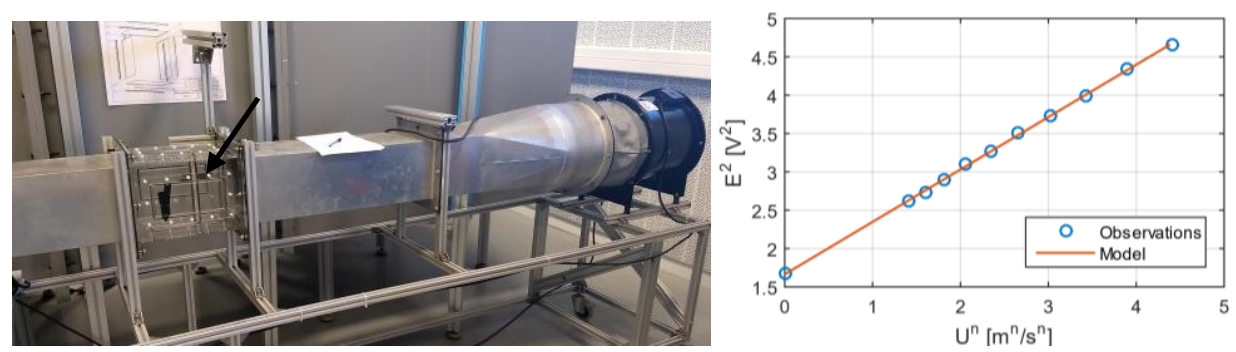

Fig. 1 Subsonic wind tunnel with cylinder installed (left) and HWA calibration curve (right)

The calibration of the hot-wire anemometer is performed by the students taking a pitot tube as a reference for the measurements and with the wind tunnel being empty (free flow conditions). Once the calibration is done, a cylinder of $23 \mathrm{~mm}$ of diameter and the same height as the measuring section (to minimize the three-dimensional effects) is introduced into the tunnel. The sensor is placed at $1.5 \mathrm{D}$ from the cylinder and then measurements are performed though the line identified in the diagram of Fig. 1 (left).

\subsection{Particle Image Velocimetry}

The Particle Image Velocimetry technique allows measuring the flow velocity through the visualization of small particles (tracer) suspended in it. For this purpose, pairs of images of these particles are compared to characterize the movement between them. A high-intensity double-pulsed Nd:YAG laser is used to illuminate the particles. The laser beam is transformed into a laser sheet by a set of cylindrical lenses. Then, a plane region is illuminated, which corresponds to the measurement region. The laser light is scattered by the particles in all directions and a camera is placed in front of the laser sheet to collect part of this radiation. As a result, bright spots patterns are registered, corresponding to the particles in the fluid. A sketch of the experimental set-up is shown in Fig. 2. 


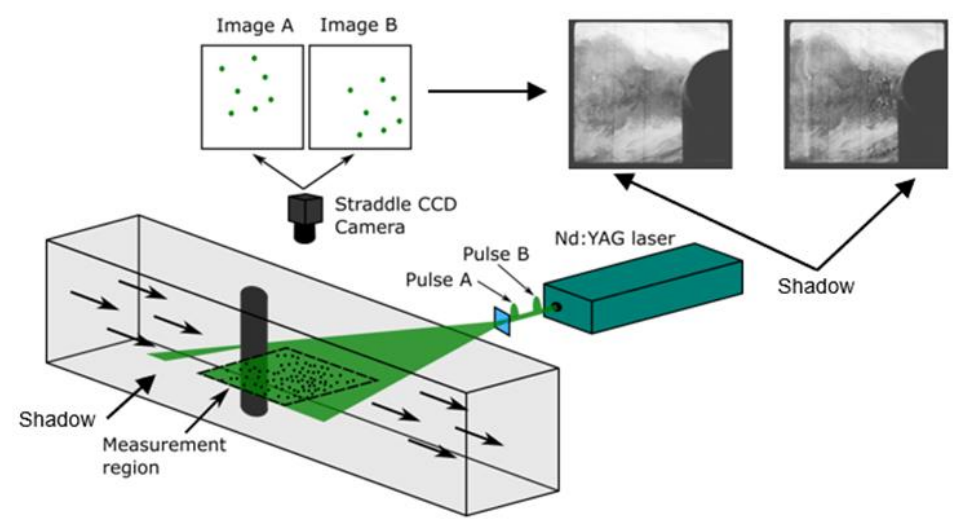

Fig. 2 Representation of the PIV experimental set-up and sample of actual images captured by the students

\section{Results and discussion}

\subsection{Sample results}

This section presents a few examples of the results obtained by students during the development of the lab session. These results will allow students to analyze the advantages and disadvantages (i.e. measurement accuracy, repeatability, etc.) of both techniques separately, and finally compare them with each other.

\subsubsection{Results of HWA measurements}

Fig. 1 (right) shows the results of the calibration procedure, one of the most critical procedures faced by experimentalist engineers. After the data processing, the relationship between flow velocity and the HWA voltage can be obtained for the whole range.

This activity helps students to familiarize themselves with the facility, to collect the first measurements and to employ advanced processing techniques to visualize the results. Fig. 3 shows a summary of the results obtained by the HWA method. The students start by plotting the measured velocities with their deviations against the number of diameters that move away from the cylinder center (left). This representation helps to visualize where there is more dispersion due to high velocities and viscous stresses. In a second step, the obtained profiles are normalized by the free-flow velocity $\left(U_{\infty}\right)$ to compare them directly (right). 
Improving the specific instrumental transversal skill of Aerospace Engineers through a lab experience


Fig. 3 Summary of the results obtained by HWA. Velocity magnitude and standard deviation (left), dimensionless velocity (right).

Here, three characteristic regions can be easily identified by the student. Both profiles are similar due to the normalization, and they show that the free-flow velocity is reached close to $1.5 \mathrm{D}$ of the center.

\subsubsection{Results of PIV measurements}

Based on PIV measurements, a comparison between PIV and HWA measurements can be performed. A velocity magnitude profile can be reproduced at the same location where HWA measurements were carried out (1.5D downstream from the cylinder location). An example of PIV measurement results is shown in Fig. 4.
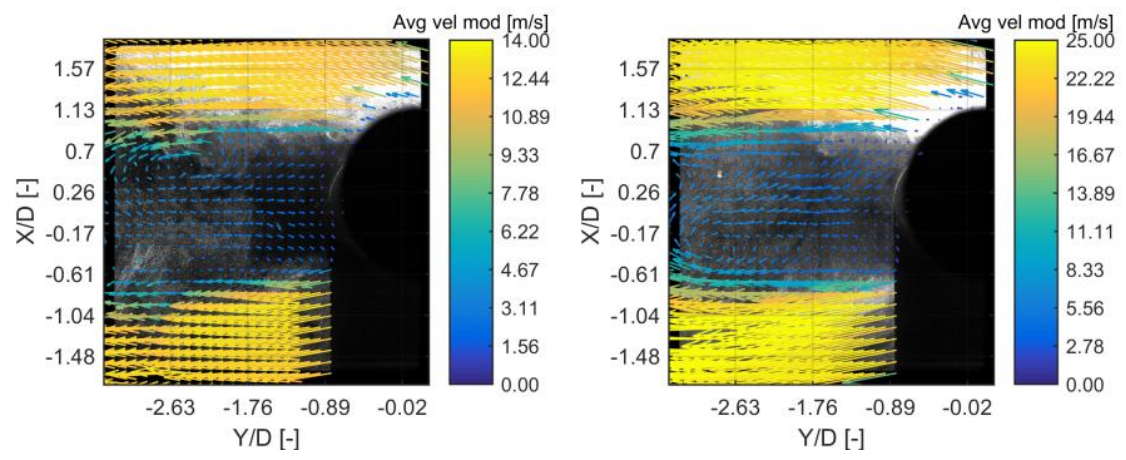

Fig. 4 PIV measurement results, measured at 10 (left) and $20 \mathrm{~m} / \mathrm{s}$ (right) free flow velocity

\subsubsection{Comparison of both techniques}

Once the two experimental techniques have been used, it is possible for the students to draw overall conclusions based on the comparison of both techniques, shown in Fig. 5. The students can observe that HWA measurements are more consistent. PIV is able to reproduce similar results, although higher speeds are predicted, probably due to slight 
maladjustment of the wind tunnel. This difference is more evident in the free-flow region, whereas the higher turbulence helps to homogenize the situation in the center of the wake.

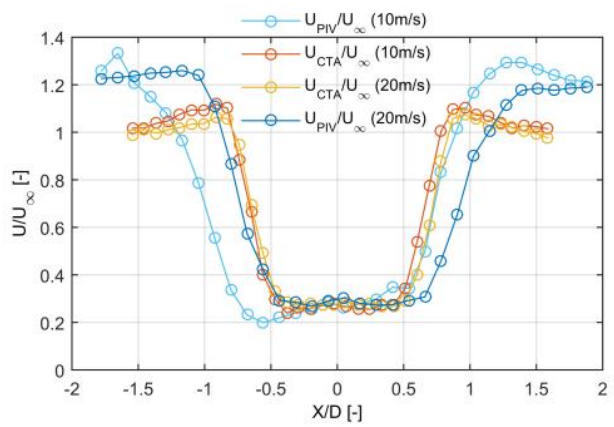

Fig.5 Overview of the results obtained by both measurement techniques.

\subsection{Outcomes}

In this section, the outcomes of the proposed work are analyzed from two points of view, in the first place the subjective opinion that the students have of the lab sessions and in the second place the objective marks that the students have been awarded.

\subsubsection{Subjective student opinion}

Student opinions are gathered at the end of the lab sessions through an anonymous poll, in which they are asked to rate different aspects of the experience (contents, resources, professor, organization, etc.) with a mark between 0 (worst) and 5 (best).

In Fig. 6 (left) the average student answers to the relevant poll question along the last four academic years is shown, demonstrating a high degree of satisfaction with the lab experience: the overall mean in the four years in 4.17/5 with an average standard deviation of 0.74 . This shows that the proposed methodology is well received by the aerospace engineering students.

\subsubsection{Objective student evaluation}

On the other hand, evaluation of the student learning in the lab experience is performed with an on-line test in which they must demonstrate the attained knowledge regarding the specific instrumentation transversal skill. This test is graded from 0 to 10 , and the average results for different academic years being shown in Fig. 6 (right), the overall mean being $7.79 \pm 0.89 / 10$. 
Improving the specific instrumental transversal skill of Aerospace Engineers through a lab experience
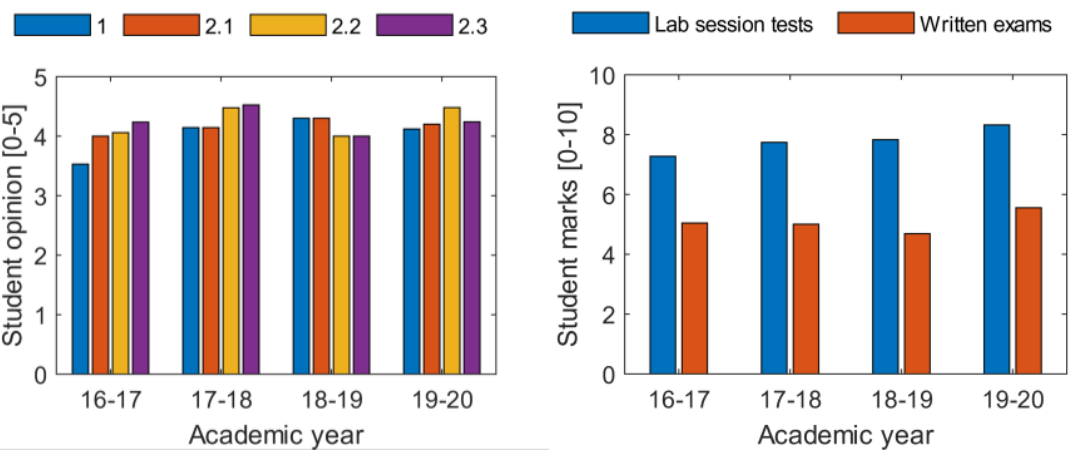

Fig.6 Average student opinion of the lab sessions (left) and average student marks on the subject (right). Student opinion key: 1-Overall opinion, 2.1-It is interesting, 2.2-It relates to the theory, 2.3-It applies the theory

In addition, the average marks of the written examns is also shown in the same plot, with an overall result of $5.07 \pm 2.25$. Year after year it can be seen that student fare remarkably better in the lab session tests, highlighting the potential of the proposed lab-based methodology to boost transversal skills acquisition in Aerospace Engineering students.

\section{Summary and conclusions}

A learning methodology for improving the specific instrumental transversal skill in a Aerospace Engineering degree course is presented in this paper. This methodology, based on an active and autonomous learning, introduces students to the field of advanced measurement techniques through practical sessions in a laboratory environment.

The students are directly involved in the complete measuring process using two of the most extended techniques to measure local fluid flow velocities. This allows the students not only to know the the specific features of each measuring method but also to identify the advantages and disadvantages of each one.

The subjective feedback from the students demonstrates that the proposed methodology is well received, evincing the high degree of satisfaction with the lab experience. The objective evaluation results show the potential benefits of the proposed lab-based methodology to boost transversal skills acquisition in Aerospace Engineering students.

\section{References}

Bloom, B. S. (1956). Taxonomy of Educational Objectives: The Classification of Educational Goals. (D. Mckay, Ed.). Ann Arbor. 
Carrillo de la Peña, M. T., \& Pérez, J. (2012). Continuous Assessment Improved Academic Achievement and Satisfaction of Psychology Students in Spain. Teaching of Psychology, 39(1), 45-47. https://doi.org/10.1177/0098628311430312

European Ministers of Education. (1999). The Bologna declaration of 19 June 1999. In The European Higher Education Area. Bologna.

Freeman, S., Eddy, S. L., McDonough, M., Smith, M. K., Okoroafor, N., Jordt, H., \& Wenderoth, M. P. (2014). Active learning increases student performance in science, engineering, and mathematics. Proceedings of the National Academy of Sciences of the United States of America, 111(23), 8410-8415. https://doi.org/10.1073/pnas.1319030111

Lapins, M. (1997). Aerospace engineering education: An industry view from a preliminary design perspective. International Journal of Engineering Education, 13(5), 376-379.

L b i B. . . \& Obig D. G. ( 7). Auh i L igf h 1 C uy: A Overview. Learning, 1(March), 1-7. $\quad$ Retrieved from http://alicechristie.org/classes/530/EduCause.pdf

Marzano, R. J., \& Kendall, J. S. (2006). The New Taxonomy of Educational Objectives. (Corwin Press - SAGE Publications, Ed.). California.

UPV. (2015). Proyecto Institucional de Competencias Transversales (in Spanish). Universitat Politècnica de València. 